\title{
Critical issues in pediatric lung transplantation
}

Forty children (aged 1 to 18 years, 27 female and 13 male) have undergone heart-lung (21), double lung (17), and single lung (2) transplant procedures at our center from 1985 through April 1994. The indications for transplantation have been diverse, primary pulmonary hypertension (10), cystic fibrosis (11), congenital heart disease (10), arteriovenous malformation (3), emphysema (1), graft-versus-host disease (1), rheumatoid lung (1), cardiomyopathy (1), desquamative interstitial pneumonitis (1), and Proteus syndrome (1). The actuarial 1-year survival was 73\% (mean follow-up 2 years). One-year actuarial survival for disease groups ranged from $60 \%$ for cystic fibrosis to $90 \%$ for congenital heart disease. We have identified six issues critical to the patient and programatic survival of pediatric lung transplantation. Our experience and management strategies in these areas are reviewed. Cytomegalovirus: Cytomegalovirus disease developed in six of eight patients with cytomegalovirus mismatching (donor + /recipient - ) and in seven of 32 patients who survived more than 30 days (23\%). All but cytomegalovirus donor -/recipient- patients were treated with ganciclovir for 4 weeks after transplantation. Obliterative bronchiolitis: Obliterative bronchiolitis developed in seven of $32(25 \%)$ patients who survived more than 30 days. Obliterative bronchiolitis was manifest within the first posttransplantation year as a rapid decline in small airway function. Aggressive augmentation of immunosuppression has been used with little success. Posttransplantation lymphoproliferative disease: Posttransplantation lymphoproliferative disease developed in five of $32(15 \%)$ patients who survived more than 30 days developed. One patient died (17\% mortality) despite retransplantation. In four patients the disease resolved with reduction in immunosuppression alone, and one required the addition of interferon alfa. Cystic fibrosis: We have changed our management strategies to avoid triple drug immunosuppression, perioperative blood and bronchial cultures, aggressive antimicrobial therapy, and exclusion of patients with panresistant organisms; this has resulted in elimination of infectious mortalities thus far in the pediatric cystic fibrosis group. Airways: In 21 heart-lung recipients with tracheal anastomoses we have had no airway complications. The double and single lung transplant recipients accounted for 34 bronchial and one tracheal anastomoses. Three (9\%) bronchial stenoses developed. Two were treated with silicone stents and one with balloon dilation. Finances: The average charge for lung transplant evaluation was $\$ 18,000$ and for transplantation, $\$ 175,000$. (J THORAC CARDIOVASC SURG 1995;109:60-5)

John M. Armitage, MD, Geoffrey Kurland, MD (by invitation), Marian Michaels, MD (by invitation), Lynne A. Cipriani, RN, BSN (by invitation), Bartley P. Griffith, MD, and Frederick J. Fricker, MD (by invitation), Pittsburgh, $P a$.

$\mathrm{P}$ ediatric lung transplantation remains in its infancy, as attested to by the St. Louis International

Read at the Seventy-fourth Annual Meeting of The American Association for Thoracic Surgery, New York, N.Y., April 24-27, 1994.

Address for reprints: John M. Armitage, MD, Associate Professor of Surgery, University of Pittsburgh Medical Center, C700 PUH-UPMC, 200 Lothrop St., Pittsburgh, PA 15213.

Copyright (C) 1995 by Mosby-Year Book, Inc.

$0022-5223 / 95 \$ 3.00+0 \quad \mathbf{1 2 / 6 / 6 0 1 3 7}$
Lung Transplant Registry, ${ }^{1}$ which has recorded nearly 2000 lung transplants performed worldwide through 1992, only $96(5 \%)$ of which were for patients between birth and 16 years of age. The International Society of Heart and Lung Transplantation's (ISHLT) 1993 Registry reported 1349 heartlung transplant procedures, only $20 \%$ of which were for patients under the age of 18 years. ${ }^{2}$ Despite the late advent of lung transplantation technology into pediatric medicine, survival rates and quality of life in these children have been gratifying. The children 


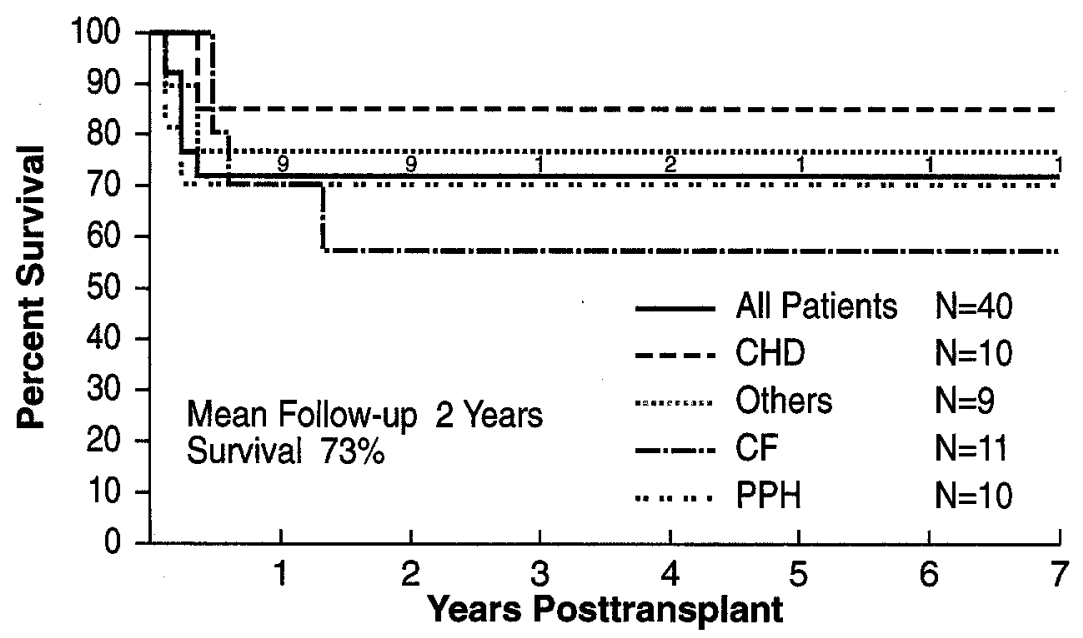

Fig. 1. Percent survival after transplantation, with the patients stratified according to their indication for transplantation. $C H D$, Congenital heart disease; $C F$, cystic fibrosis; $P P H$, primary pulmonary hypertension.

are clearly beneficiaries of some very difficult lessons learned in the adult arena. However, as always, the children have much to teach us. We report here our experience in pediatric lung transplantation with emphasis on issues critical to the management and long-term well-being of these special patients.

\section{Patient group}

Forty children, aged 1 to 18 years, have undergone heart-lung $(n=21)$, double lung $(n=17)$, and single lung $(n=2)$ transplantation from 1985 through April 1994. Twenty-eight $(70 \%)$ of these children were female and 12 were male. The indications for transplantation have been primary pulmonary hypertension $(n=10)$, cystic fibrosis (CF) $(n=11)$, congenital heart disease $(n=10)$, arteriovenous malformation $(n=3)$, emphysema $(n=1)$, graft-versus-host disease $(n=1)$, rheumatoid lung $(n=$ $1)$, cardiomyopathy $(n=1)$, desquamative interstitial pneumonitis $(n=1)$, and Proteus syndrome $(n=1)$. The congenital heart anomalies in our 10 patients were transposition of the great arteries $(n=5)$, ventricular septal defect $(n=2)$, truncus arteriosus $(n=1)$, hypoplastic left heart syndrome $(n=1)$, and double-outlet right ventricle $(n=1)$. Nine $(22 \%)$ of the 40 recipients had at least one major thoracic operation before transplantation. Two of the nine patients had two sternotomies, one had two thoracotomies, and one had two sternotomies and two thoracotomies. One patient underwent retransplantation for posttransplantation lymphoproliferative disease (PTLD).

A review of our center's recipient selection, implantation techniques, donor evaluation, and lung preservation, immunosuppression, monitoring and treatment of infection and rejection has been previously reported. . $^{3,4}$

Survival. Actuarial survival at 1 year for the group as a whole was $73 \%$ at a mean follow-up of 2 years (range 0.5 to 8 years). One-year actuarial survival for individual disease groups is depicted in Fig. 1 and ranged from a low of $60 \%$ for patients with CF to $90 \%$ for patients with congenital heart disease (CHD).

Critical issues. We have chosen six issues critical to survival in pediatric lung transplantation, both to the individual patients and to the programs themselves. Our experience and management strategies in these areas are reviewed herein.

Cytomegalovirus (CMV). Thirty-two patients survived more than 30 days after transplantation. CMV disease, defined as the presence of CMV syndrome, pneumonitis, hepatitis, or enteritis, developed in seven patients $(23 \%)$. Donor (D) and recipient (R) CMV serology was available on 30 of these 32 patients. Nineteen patients were $\mathrm{D}-1$ $\mathrm{R}-$, and CMV disease developed in only one of these patients. This occurred in 1985, before the advent of ganciclovir, routine donor/recipient CMV screening, and the exclusive use of CMV negative blood products for transplant recipients. CMV pneumonitis developed after discharge on posttransplantation day 20 , and the patient subsequently died of disseminated CMV. This has been our only CMV mortality, undoubtedly the result of blood product transmission.

Eight patients were $\mathrm{CMV}$ mismatched, $\mathrm{D}+/ \mathrm{R}-$, and CMV disease developed in six $(75 \%)$ of them. CMV pneumonitis developed in four patients and CMV syndrome developed in two. One patient was $D-/ R+$ and two patients were $\mathrm{D}+/ \mathrm{R}+$; no $\mathrm{CMV}$ disease developed in these patients; however, CMV shedding was detected in their bronchial lavage samples.

Our present approach to the issue of CMV in pediatric lung transplant recipients includes the following:

1. Donor and recipient serologies are always obtained and do not affect donor or recipient selection. CMV serology is part of the routine screening of all lung recipients at their semiannual follow-up visits. In addition, bronchoalveolar lavage fluid and lung biopsy samples are routinely screened for CMV. 
2. All lung transplant recipients who require transfusions receive CMV negative blood and blood products.

3. All CMV positive recipients or mismatched recipients $(D+/ R-)$ receive intravenous ganciclovir for a minimum of 4 weeks $(10 \mathrm{mg}$ per $\mathrm{kg}$ per day in two divided doses for 2 weeks and $5 \mathrm{mg} / \mathrm{kg}$ per day once a day for 2 weeks). In the past, we have recommended 3 months of high-dose $\left(650 \mathrm{mg} / \mathrm{m}^{2}\right.$ per day) oral acyclovir to these patients; however, a randomized prospective study in pediatric liver recipients has shown this to be of no added benefit in preventing CMV disease. In addition, pharmacokinetic studies have revealed both very poor absorption of acyclovir in children and correspondingly low serum levels. ${ }^{5}$ We have, therefore, discontinued this practice.

4. The recent availability of CMV-specific immunoglobulin carries with its hope of passive CMV immunity a very high price tag. We intend to examine its efficacy as an adjuvant to ganciclovir in our high-risk population in our next five patients. We have not previously used polyclonal immunoglobulin preparations.

Obliterative bronchiolitis $(O B)$. In our 32 patients who survived more than 30 days, an average of two episodes of acute cellular rejection (A2A or greater) occurred per patient within 90 days, irrespective of the primary immunosuppressant, cyclosporine or FK 506 . However, from 90 days to 2 years, the FK 506 group had significantly less acute cellular rejection (2.2 versus 0.4 rejection episodes per patient) than the cyclosporine group $(p<0.001$, Fisher). OB developed in five patients (30\%) in the cyclosporine group and two (12\%) in the FK 506 group. The incidence of $\mathrm{OB}$ in the pediatric lung transplant group as a whole was $25 \%$. Three $(40 \%)$ of the seven children with $\mathrm{OB}$ had CMV disease and all were CMV negative recipients. All seven children with OB demonstrated a dramatic and often sudden drop in small airway function as measured by forced expiratory volume in 1 second and maximum midexpiratory flow rate within the first posttransplantation year. Only one patient has shown significant improvement in airway function with aggressive augmentation of immunosuppression. Our treatment protocol for $\mathrm{OB}$ is two courses of intravenous steroids and at least one course of antilympholytic therapy.

This aggressive approach has yielded little success. Inhaled cyclosporine, total lymphoid irradiation, and photophoresis are all potential treatment options focused on further augmentation of immunosuppression. All of these therapies would have to be considered experimental at this time, and although we have limited experience with inhaled cyclosporine in adults (10 patients) and in children (one patient), one child with OB recently underwent successful retransplantation.

PTLD. In a 10-year retrospective analysis of heart and lung transplantation with cyclosporine-based immunosuppression, we found that PTLD was significantly more likely to develop within the first posttransplantation year in the lung transplant recipient than in the heart recipient ( $7.9 \%$ versus $1.8 \%, p=0.02$ ). In the same study it was apparent that the child was at much greater risk than the adult for the development of PTLD (9.7\% versus $3.5 \%$, $p=0.08){ }^{6}$ Thus it has not surprised us that PTLD developed in $15 \%$ of our pediatric lung transplant recipient group who survived more than 30 days. Five children have had PTLD; in all the disease became manifest in the lung within the first year after transplantation, and all were Epstein-Barr virus (EBV) negative recipients. One patient $(17 \%)$ died despite retransplantation. Three patients were rendered disease free with reduction in immunotherapy alone, and the fourth required the addition of interferon alfa for remission. Ten other children were EBV negative recipients, two had EBV syndrome, and one had EBV hepatitis without PTLD.

In addition to maintaining a high index of suspicion for this disease, we now also obtain donor and recipient EBV serology. In the event of an EBV mismatch, $\mathrm{D}+/ \mathrm{R}-$, we reduce immunosuppression to single-agent therapy (FK 506 or cyclosporine) and aggressively monitor EBV serology in the recipient's serum, as well as special EBV stains and probes of lung biopsy specimens. We have reserved the use of interferon alfa for patients in whom computed tomographic scanning of the chest fails to demonstrate significant resolution of PTLD lesions in the lung within 4 to 8 weeks after their reduction in immunotherapy. We also use both ganciclovir intravenously and acyclovir orally during the early course of PTLD. However, data to suggest that this is truly beneficial are scant. One of our four children who obtained remission from PTLD had recurrence 5 years later with cervical adenopathy and EBV syndrome. She has continued to do well receiving reduced immunosuppression.

$C F$. The 1993 ISHLT Registry reported that CF was the indication for double lung transplantation in more than $60 \%$ of children undergoing the procedure. Another $18 \%$ of children who underwent heart-lung transplantation also had CF. Thus not only has CF become the leading indication for lung transplantation in children, it carries the highest risk. One-year survival after doublelung transplantation for $\mathrm{CF}$ is approximately $60 \%$. Along with the problems associated with all other patients with end-stage lung disease, patients with CF have tracheal colonization with organisms that are difficult to treat with antibiotics. Patients with CF also commonly are malnourished and have chronic sinusitis and osteoporosis. Indwelling intravenous or enteral catheters are frequently in place at the time of transplantation.

In our entire patient group, we had 31 major infections: Ten were bacterial, and $80 \%$ occurred in patients with CF; eight were viral, and $50 \%$ occurred in patients with CF; two were yeast, and $100 \%$ occurred in patients with CF. Seven patients died of infection (11), four $(60 \%)$ of whom had CF. We have learned from our patients with $\mathrm{CF}$ that infection poses their greatest risk, and we have thus altered our approach to them, such that our last four patients with CF have all been discharged without a major infection after double lung transplantation.

1. The presence of Pseudomonas cepacia in the airway or sinuses of a patient with $\mathrm{CF}$ is a contraindication to lung transplantation.

2. Blood cultures are obtained during the transplant procedure and in the immediate postoperative period. These cultures are positive for Pseudomonas in about $50 \%$ of CF recipients. Early identification and sensitivity of blood-borne organisms is tremendously helpful in directing specific and aggressive antibiotic therapy. Antibiotics include an aminoglycoside and at least one other agent 
directed against the patient's organisms. Amphotericin is used aggressively for any indication of yeast colonization. This antibiotic regimen is continued initially for a minimum of 2 weeks. Bronchoscopy and bronchoalveolar lavage are done routinely within 24 to 48 hours after transplantation to further identify organisms that may have colonized the transplanted airway in the early postoperative period.

3. Triple-drug immunosuppression is avoided now in all recipients with $\mathrm{CF}$. They receive steroids only during the operation and on postoperative day 1. Thereafter, FK 506 (or cyclosporine) and azathioprine are the only immunosuppressive agents.

4. We do not recommend sinus ablation before or after transplantation. This has not been of specific benefit and is attended with recolonization within 6 months. Furthermore, we have not documented that sinusitis per se has been a significant contributing factor to posttransplantation infections in our patients with CF.

Airways. We use polypropylene sutures for all airway anastomoses. We no longer use omental or vascularized pedicle wraps for any airway anastomosis. In heart-lung recipients, we do interpose a strip of pericardium between the trachea and aorta to provide some barrier to infectious organisms that might lead to an aortic mycotic aneurysm. We have not as yet pursued techniques designed specifically to revascularize the airway. All our heart-lung transplants in children are performed with end-to-end tracheal anastomoses just above the carina. This is done with a running 3-0 polypropylene suture on the membranous portion and a separate running suture on the cartilaginous portion. In 20 heart-lung recipients, we have had no airway complications and no aortic mycotic aneurysms. Only one double lung recipient had a tracheal anastomosis; all other double lung recipients and both single lung recipients had bronchial anastomoses. We perform our bronchial anastomoses with a running polypropylene suture on the membranous portion and interrupted figureof-eight telescoping sutures on the cartilaginous portion. We have encountered one airway anomaly, a tracheal origin of the right upper lobe bronchus. ${ }^{7}$ The double and single lung recipients accounted for 34 bronchial and one tracheal anastomoses. There were three (9\%) airway stenoses in the 34 bronchial anastomoses. Two were treated with a bronchial stent and one with balloon dilation.

Finances. The average charge for a lung transplant evaluation at Children's Hospital of Pittsburgh, which usually involves a 72 -hour admission, is $\$ 18,000$ (range $\$ 12,000$ to $\$ 28,000)$. The average charge for a lung transplant procedure and 3 weeks' hospitalization is $\$ 175,000$ (range $\$ 150,000$ to $\$ 325,000$ ).

\section{Discussion}

Despite the fact that $\mathrm{CF}$, congenital heart disease, and primary pulmonary hypertension constitute the main indications for lung transplantation in children, a wide variety of end-stage lung diseases in children can be successfully treated by transplantation. The survival statistics from our own and many other centers have been extremely gratifying. However, those of us involved in this challenging endeavor remain far from the summit of success. The exhilaration, for both physician and child alike of the restoration toward normality of heart and lung function after transplantation defies description. Having once experienced this thrill, nothing is more agonizing than to watch the breathlessness of $\mathrm{OB}$ ensue. It is incumbent on all of us involved in this field to pursue without respite a better understanding of the immunologic factors that lead to this devastating manifestation of chronic lung rejection. It seems, with the rapid onset of $\mathrm{OB}$, that additive and more potent immunosuppression as a means of treatment is too little too late. We must be able to better understand the disease, predict those at risk, and devise a strategy of prevention. The clues to the puzzle of $\mathrm{OB}$ lie in the determination of why the bronchial epithelium is the antigenic target and what cytokines up-regulate either humoral or cellular components to such a destructive end. Indeed, although CMV deserves further study and understanding to prevent its disease, there is much evidence to suggest that it plays a role in immune up-regulation and therefore may be a significant factor in the development of $\mathrm{OB}^{8}{ }^{8}$

PTLD also remains a major concern to the pediatric organ recipient, particularly if the transplanted organ contains significant amounts of donor-derived lymphatic tissue, as in lung and small bowel. We are convinced that, at least for those patients in whom PTLD develops early after transplantation, PTLD is a donor-transmitted disease. EBV serology of donor and recipient is as important as is that of CMV, particularly in the child. Early detection and aggressive reduction in immunotherapy are keys to the successful treatment and management of PTLD. This disease underscores the critical importance of using and administering immunosuppressive agents, not in a "cook book" fashion, but individualized to each patient.

Another significant challenge in pediatric lung transplantation is to improve the results in our largest patient population, that of CF. Ideally, in the near future genetic engineers will obviate the need for lung replacement therapy in these children. However, until such time, we must recognize that infection poses the greatest risk to lung recipients with $\mathrm{CF}$ and that lower immunosuppression and aggressive antimicrobial diagnosis and therapy will improve the outcome.

Finally, we the transplant physicians must address 
the issue of cost. This is a painful and arduous task. Innovative and fiscally sound decisions must be made in a multiinstitutional and cooperative effort to hold down and limit the costs involved in this astronomically expensive procedure.

\section{REFERENCES}

1. St. Louis International Lung Transplant Registry, April 1993 Report.

2. The Registry of the International Society for Heart and Lung Transplantation: Tenth Official Report-1993. Compiled by Michael P. Kaye, MD, with the cooperation of the 229 cooperating centers. J Heart Lung Transplant 1993;12:541-8.

3. Armitage JM, Fricker FJ, Kurland G, et al. Pediatric lung transplantation: the years 1985 to 1992 and the clinical trial of FK 506. J THORAC CARDIOVASC SURG 1993;105:337-46.

4. Armitage JM, Fricker FJ, Kurland G, Michaels M, Griffith BP. Pediatric lung transplantation: expanding indications: 1985-1993. J Int Soc Heart Lung Transplant [In press].

5. Green M, Reyes J, Nour B, et al. Randomized trial of ganciclovir (GAN) followed by high-dose oral acyclovir (HD-ACY) vs gan alone in the prevention of cytomegalovirus (CMV) disease in pediatric liver transplant (PLTx) recipients. Transplant Proc [In press].

6. Armitage JM, Kormos RL, Stuart RS, et al. Posttransplant lymphoproliferative disease in thoracic organ transplant patients: ten years of cyclosporine-based immunosuppression. J Heart Lung Transplant 1991;10: 877-86.

7. Magee MJ, Griffith BP, Armitage JM. Successful management of a right tracheal bronchus in a pediatric double lung transplant recipient. Ann Thorac Surg [In press].

8. Keenan RJ, Lega ME, Dummer JS, et al. Cytomegalovirus serologic status and postoperative infection correlated with risk of developing chronic rejection after pulmonary transplantation. Transplantation 1991; 51:433-8.

\section{Discussion}

Dr. J. Kent Trinkle (San Antonio, Tex.). Are you accepting mismatches-CMV positive donor organs into CMV negative patients?

Dr. Armitage. Correct. We do not direct organs to CMV status and we will accept a mismatch.

Dr. Trinkle. Am I correct that you use only ganciclovir for prophylaxis?

Dr. Armitage. That's correct. In the past we have used intravenous ganciclovir for 4 weeks.

Dr. Trinkle. In the past we have had terrible problems with CMV, particularly with cytolytic therapy and specifically with OKT3. We then started using both prophylactic ganciclovir and immune globulin. The lung transplant literature contained nothing in this regard, but a fair amount of data from other transplant systems and bone marrow transplants indicated that the combination of the two is better than just one.

Dr. Armitage. We have avoided, except early on, the use of cytolytic therapy in children specifically because of the increased risk of CMV and also EBV.

Dr. Thomas M. Egan (Chapel Hill, N.C.). If you are going to bring up the issue of cost of transplantation, I think it is only fair to bring up the issue of cost of care of chronically ill children. Do you have any idea what the cost of care would have been until these children died without transplantation, because that is the other side of the equation?

Dr. Armitage. The cost/benefit ratio is certainly an excellent point. Most of the children that we deal with have a fairly short life expectancy. However, the patient with CF can live for many years, and the cost is tremendous.

Dr. Axel Haverich (Kiel, Germany). Something worrisome is the very high rate of $\mathrm{OB}$ in the pediatric age group. Were you able to identify special risk factors, or could you identify certain immunosuppressive regimens, such as FK 506, to have a beneficial effect on this complication?

Dr. Armitage. The recognition and prevention of $O B$ is probably the crux of the matter. In the larger population of adults, two major risk factors have been identified by multivariate analysis. The first is the frequency of acute cellular rejection as a major risk factor-more than two or three episodes in the first 3 months is a risk factor for the development of OB. The other factor is the development of $\mathrm{CMV}$ viral infection. Of 10 adult recipients who eventually had $\mathrm{OB}$, nine had prior CMV infections. $\mathrm{OB}$ is much less prevalent in those who do not have CMV infections. Thus CMV and acute cellular rejection are the main issues.

Dr. Haverich. If you evaluate your adult population, does FK 506 make any difference?

Dr. Armitage. Episodes of acute cellular rejection are less frequent in adults, and that may affect $\mathrm{OB}$, but we have certainly not seen an effect in the pediatric group.

Dr. Vaughn A. Starnes (Los Angeles, Calif.). What are you doing now with Eisenmenger's syndrome in your pediatric age group?

Dr. Armitage. All 10 of these children underwent heart-lung transplantation, and even in retrospect, none of them probably would have been good candidates for single or double lung transplantation. They are a very special group, as you know. Many of them have had three or four prior procedures, and most of the cardiac defects in these younger children have been irreparable. We have had repairs of atrial septal defects, ventricular septal defects, and atrioventricular septal defects when the leftsided atrioventricular valve was reparable. Patent ductus arteriosus and transposition of the great arteries have been repaired concomitantly with double lung transplantation. A small number of children with atrial septal defects have undergone single lung transplantation. We do have one child with a ventricular septal defect who I think is a candidate for double lung transplantation rather than heart-lung transplantation.

Dr. Trinkle. What do you use for bronchial stenoses? 
We use the Palmaz stent, which was developed by Dr. Julio Palmaz, a vascular radiologist at our institution, and is made by Johnson \& Johnson (King of Prussia, Pa.). It is a wire mesh vascular stent, such as you use in coronary or femoral arteries. It is available in various lengths and sizes and is very easy to put in. I highly recommend it.

Dr. Armitage. We have used silicone rubber stents. I know that Chip Bolman at the University of Minnesota has used chicken wire stents, and I think they have a lot of appeal. If the right upper lobe bronchus is very near, you can continue to ventilate and stent it. We used balloon dilation in one patient in whom the right upper lobe bronchus was right there, but I think that the mesh stent certainly has a role.

\section{Availability of JourNaL back issues}

As a service to our subscribers, copies of back issues of THE Journal of THORACIC AND CARDIOvasCULAR SURGERY for the preceding 5 years are maintained and are available for purchase from the publisher, Mosby-Year Book, Inc., at a cost of $\$ 12.00$ per issue. The following quantity discounts are available: $25 \%$ off on quantities of 12 to 23 , and one third off on quantities of 24 or more. Please write to Mosby-Year Book, Inc., Subscription Services, 11830 Westline Industrial Drive, St. Louis MO 63146-3318, or call (800)453-4351 or (314)453-4351 for information on availability of particular issues. If unavailable from the publisher, photocopies of complete issues are available from University Microfilms International, 300 N. Zeeb Rd., Ann Arbor, MI 48106, (313)761-4700. 